\title{
Connections between millimetre continuum variations and VLBI structure in 27 AGN
}

\author{
T. Savolainen ${ }^{1}$, K. Wiik ${ }^{1,2}$, E. Valtaoja ${ }^{1,3}$, S. G. Jorstad ${ }^{4}$, and A. P. Marscher ${ }^{4}$ \\ 1 Tuorla Observatory, Väisälä Institute for Space Physics and Astronomy, University of Turku, \\ 21500 Piikkiö, Finland \\ 2 Metsähovi Radio Observatory, Helsinki University of Technology, 02540 Kylmälä, Finland \\ 3 Department of Physics, University of Turku, 20100 Turku, Finland \\ ${ }^{4}$ Institute for Astrophysical Research, Boston University, 725 Commonwealth Ave., Boston, MA 02215, USA
}

Received 14 May 2002 / Accepted 27 August 2002

\begin{abstract}
We compare total flux density variations in $27 \gamma$-ray blazars with structural changes in their parsec-scale jets using multi-epoch VLBA observations at 22 and $43 \mathrm{GHz}$ together with data from the Metsähovi quasar monitoring program at 22 and $37 \mathrm{GHz}$. There is a clear connection between total flux density outbursts and VLBI components emerging into the jet. For essentially every new moving VLBI component, there is a coincident total flux density flare, with evolution similar to that of the component. Furthermore, extrapolated ejection times of the new VLBI components correspond to the beginnings of associated flares. Our results suggest that it is possible to explain all the radio variations as shocks propagating down the jet. A large fraction of the shocks grow and decay within the innermost few tenths of a milliarcsecond and therefore we see them only as "core flares" in the VLBI images. However, with present data we cannot exclude the possibility that the core itself also brightens (and thus contributes to the flare) as a shock passes through it.
\end{abstract}

Key words. BL Lacertae objects: general - galaxies: active - galaxies: jets - quasars: general - radio continuum: galaxies techniques: interferometric

\section{Introduction}

Blazars are an interesting and violent subclass of active galactic nuclei (AGN), grouping together (although somewhat artificially from a physical point of view) radio-loud quasars and BL Lacertae objects. These sources have in common flat $\mathrm{cm}$-wave radio spectrum, high and variable polarization, and pronounced variability of the flux density at all frequencies. The superluminal motion observed in these sources together with brightness temperatures in excess of the $10^{12} \mathrm{~K}$ inverse Compton limit (Kellermann \& Pauliny-Toth 1968) indicate highly beamed emission from relativistic jets oriented towards the line of sight of the observer. Using modern-day very long baseline interferometry (VLBI) techniques we can resolve the jet-like structures in blazars on angular scales down to $\sim 0.1$ milliarcsec (mas).

Relativistic jets also offer an explanation for radio-toinfrared variability of blazars. Marscher \& Gear (1985) studied the strong 1983 outburst in 3C 273 and managed to fit the flaring spectra with self-absorbed synchrotron emission. They explained successfully the time-evolution of the flare as being due to a shock wave propagating in the relativistic jet.

Send offprint requests to: T. Savolainen, e-mail: tukasa@astro.utu.fi
The Marscher \& Gear model (hereafter MG-model) has three stages of shock evolution based on the dominant cooling mechanisms of the electrons: 1) the Compton scattering loss phase, 2) the synchrotron radiation loss phase and 3) the adiabatic expansion loss phase. MG-model is a simple, analytical model, which describes well the general behaviour of the radio outbursts in AGN (but see the critíque of Björnsson \& Aslaken 2000). The model was generalized by Marscher et al. (1992) to include the effects of bending in jets and turbulence on the light curves.

Hughes et al. (1985, 1989a, 1989b, 1991) proposed a similar shock model based on a numerical code simulating a pistondriven shock. Their model succesfully explains the lower frequency variability, but it does not incorporate radiative energy losses of the electrons, which are important at high frequencies and in the earliest stages of the shock evolution. Valtaoja et al. (1992b) presented a generalized shock model describing qualitatively the three stages of the shock evolution (growth, plateau and decay) without going into details, thus providing a framework for comparison between the theory and observations. Total flux density (TFD) monitoring campaigns, which provide nearly fully sampled flux curves at radio wavelengths, and VLBI images, which allow us to map the parsec-scale 
structure of the blazar jets, are the two main observational tools for constraining theoretical models.

In VLBI observations of blazars, bright knots of emission referred to as "components" are seen. These components line up to form jet-like features appearing in various forms from very straight to heavily bent structures. The so-called "core" is the point where the jet becomes visible. The core is presumed stationary (see Bartel et al. 1986), but most of the other VLBI components move outward in the jet at apparent superluminal speeds. However, in some sources there are also stationary components other than the core. These may be due, for example, to interactions between the jet and the surrounding interstellar medium.

According to the shocked jet models, moving components in the VLBI maps are interpreted as shocks propagating down the jet. However, there has been a dearth of conclusive evidence linking VLBI components with radio flux variations; only a relatively small number of individual sources have been investigated thus far. One of the first studies linking TFD variations with moving knots in the VLBI maps was carried out by Mutel et al. (1990). They found that each of four major TFD outbursts of BL Lac between 1980 and 1988 can be associated with the emergence of a new superluminal component. Abraham et al. (1996) estimated the ejection times of seven VLBI components in 3C 273 and noticed that all ejections were related to increases in the single-dish flux density at frequencies higher than $22 \mathrm{GHz}$. Türler et al. (1999) also studied 3C 273 by decomposing multi-frequency light curves into a series of self-similar flares. They found good correspondence between the ejection times of the VLBI components and the beginning times of the flares. Krichbaum et al. (1998) have reported a correlation between mm-VLBI component ejections and local minima in the $90 \mathrm{GHz}$ total flux density curve of PKS 0528+134. For 3C 345, which is one of the best observed sources with VLBI at $22 \mathrm{GHz}$, Valtaoja et al. (1999) were able to associate VLBI components with individual millimetre flares. Similar correlations were also found for PKS 0420-014 (Britzen et al. 2000) and for 3C 279 (Wehrle et al. 2001).

In our study, we compare for the first time two large data sets: multi-epoch VLBA images of 42 blazars (Jorstad et al. 2001a) detected at $0.1-3 \mathrm{GeV}$ by EGRET and TFD data from the mm-wave Metsähovi Radio Observatory quasar monitoring program. A description of our data is given in Sect. 2. Our aim is to establish connections between TFD variations and structural changes in the jets. The results from the analysis, as we will show in Sect. 3, strongly support the shocked jet model.

The VLBI core is the dominant component in almost all the cases studied. The core region was usually also highly variable, being responsible for most of the observed TFD variability in these sources. Variations in the VLBI core flux are reported in the literature quite often (see, e.g., the results of the recent VLBA monitoring of 3C 279 by Wehrle et al. 2001). Since the core is usually assumed to be the apex of the jet, the implicit assumption is that a core flare results from a change in the jet flow parameters. However, according to our study, these variations are rather related to moving VLBI components that blend with the radio core. This will be discussed in Sect. 4 .

\section{The data}

Our data set consists of observations from two separate campaigns, namely the VLBA monitoring of EGRET-detected blazars by Jorstad et al. (2001a) and the Metsähovi Radio Observatory quasar monitoring program. Jorstad et al. (2001a) monitored a sample of $42 \gamma$-bright blazars at 22 and $43 \mathrm{GHz}$ with VLBA between 1993 and 1997. For 27 of these sources (see Table 1), there were enough TFD variation data available from Metsähovi monitoring (Teräsranta et al. 1998) to reliably identify large outbursts in the flux curves.

Selection criteria for sources observed by Jorstad et al. (2001a) with the VLBA were: (1) detection by EGRET ( $E>$ $100 \mathrm{MeV}$; Hartman et al. 1999); (2) flux density at $37 \mathrm{GHz} \gtrsim$ $1 \mathrm{Jy}$; and (3) declination $(\mathrm{J} 2000) \geq-30^{\circ}$. These criteria give a sample containing roughly $60 \%$ of the known $\gamma$-ray blazars (Hartman et al. 1999). The VLBA observations were made at high radio frequencies giving $\approx 0.1-0.3$ mas resolution and $\approx 10$ mas map size. High resolution allows us to study the inner parts of the jet and possibly see how the shock formation is connected to the flaring behaviour of these sources. However, as we discuss below, VLBA maps at $43 \mathrm{GHz}$ in many cases still have insufficient resolution to separate the new shock in the jet from the core before the millimetre flare is over.

Since its beginning in 1980, the Metsähovi quasar monitoring program has been the most comprehensive such program at high radio frequencies. The Metsähovi sample contains 157 individual sources including about 100 of the brightest radio-loud AGN in the Northern hemisphere (declination $\approx-10^{\circ}$ ), which are observed at 22,37 and $87 \mathrm{GHz}$ (see Teräsranta et al. 1998 for details). The sample also includes the Northern 2 Jy catalogue of flat spectrum sources (Valtaoja et al. 1992a) fulfilling the following criteria: $\delta \geq 0^{\circ}, \alpha_{\text {Kuhr }}(2.7-$ $5 \mathrm{GHz}) \geq-0.5 \quad\left(S \propto v^{\alpha}\right)$, with $\alpha$ taken from the catalogue of Kühr et al. (1981), and $S_{\max }(22 \mathrm{GHz}) \geq 2 \mathrm{Jy}$. Of the 27 sources in our study, 13 belong to this 2 Jy catalogue. Of the 14 sources that do not belong, 5 have declination below $0^{\circ}$ and the rest were fainter than $2 \mathrm{Jy}$ at $22 \mathrm{GHz}$ prior to 1992 . In our study, we used 22 and $37 \mathrm{GHz}$ Metsähovi data from 1990 to 1998 together with 22 and $43 \mathrm{GHz}$ VLBA maps. Comparing $37 \mathrm{GHz}$ TFD-data with $43 \mathrm{GHz}$ VLBA maps is justified by the typically flat spectra of our sources in the millimetre region.

Our sample of 27 sources with good VLBI and TFD data consists of 12 high optical polarization quasars (HPQs), 7 low optical polarization quasars (LPQs), 7 BL Lacertae objects (BLOs) and one object classified as a radio galaxy (GAL). The percentage of each class of radio-loud AGN is given in Table 2 for our sample, for the 2 Jy sample (Valtaoja et al. 1992a), and for a sample containing the EGRET blazar identifications that have a high probability of being correct (Mattox et al. 2001). As one can see, our sample is very similar to the $\gamma$-ray blazars as well as to the 2 Jy sample representing the brightest radioloud AGN. [The one radio galaxy in our sample, $0446+112$, is a less certain EGRET identification, which is the reason why it is not included in the list by Mattox et al. (2001).] This supports the notion that the results presented in this paper are applicable to all $\gamma$-ray blazars and, to some extent, to radio-loud AGN in general. 
Table 1. List of sources in our sample. Here $z$ is the redshift and $N$ is the total number of VLBA observations.

\begin{tabular}{lllccccc}
\hline \hline Source & Other desig. & Class & $z$ & Epochs & $22 \mathrm{GHz}$ & $43 \mathrm{GHz}$ & $N$ \\
\hline $0202+149$ & & HPQ & 0.833 & $1995-97$ & - & + & 4 \\
$0219+428$ & 3C 66A & BLO & 0.444 & $1995-97$ & + & + & 7 \\
$0234+285$ & CTD 20 & HPQ & 1.207 & $1995-97$ & + & - & 4 \\
$0235+164$ & AO 0235+164 & BLO & 0.94 & $1995-96$ & - & + & 6 \\
$0420-014$ & OA 129 & HPQ & 0.915 & $1995-97$ & + & + & 8 \\
$0446+112$ & & GAL & 1.207 & $1995-97$ & - & + & 4 \\
$0458-020$ & & HPQ & 2.286 & $1995-97$ & - & + & 5 \\
$0528+134$ & & LPQ & 2.07 & $1994-97$ & + & + & 11 \\
$0716+714$ & & BLO & $>0.2$ & $1995-97$ & + & - & 9 \\
$0804+499$ & OJ 508 & HPQ & 1.43 & $1996-97$ & + & - & 3 \\
$0827+243$ & & LPQ & 2.046 & $1995-97$ & + & + & 6 \\
$0836+710$ & & HPQ & 2.17 & $1995-97$ & + & + & 7 \\
$0851+202$ & OJ 287 & BLO & 0.306 & $1995-96$ & - & + & 7 \\
$0954+658$ & & BLO & 0.367 & $1995-96$ & + & - & 3 \\
$1101+384$ & Mkn 421 & BLO & 0.031 & $1995-97$ & + & - & 8 \\
$1156+295$ & 4C 29.45 & HPQ & 0.729 & $1995-97$ & + & - & 5 \\
$1219+285$ & ON 231 & BLO & 0.102 & $1995-97$ & + & - & 3 \\
$1222+216$ & 4C 21.35 & LPQ & 0.435 & $1996-97$ & + & - & 2 \\
$1226+023$ & 3C 273 & LPQ & 0.158 & $1993-95$ & + & + & 5 \\
$1253-055$ & 3C 279 & HPQ & 0.538 & $1993-97$ & + & + & 10 \\
$1510-089$ & & HPQ & 0.361 & $1995-97$ & - & + & 5 \\
$1606+106$ & & LPQ & 1.226 & $1994-97$ & + & + & 5 \\
$1611+343$ & DA 406 & LPQ & 1.401 & $1994-97$ & + & + & 11 \\
$1633+382$ & 4C 38.41 & LPQ & 1.814 & $1994-96$ & + & - & 6 \\
$1741-038$ & & HPQ & 1.054 & $1995-97$ & + & - & 2 \\
$2230+114$ & CTA 102 & HPQ & 1.037 & $1995-97$ & - & + & 7 \\
$2251+158$ & 3C 454.3 & HPQ & 0.859 & $1995-96$ & - & + & 12 \\
\hline
\end{tabular}

\section{Connections between millimetre flux curves and VLBI components}

In order to compare TFD and VLBI events in our data, we decompose the total flux density variations in the Metsähovi $22 / 37 \mathrm{GHz}$ flux curves into exponential flares of the form

$\Delta S(t)= \begin{cases}\Delta S_{\max } \mathrm{e}^{\left(t-t_{\max }\right) / \tau}, & t<t_{\max } \\ \Delta S_{\max } \mathrm{e}^{\left(t_{\max }-t\right) / 1.3 \tau}, & t>t_{\max }\end{cases}$

Here $\Delta S_{\max }$ is the maximum amplitude of the flare, $t_{\max }$ is the epoch of the flare maximum and $\tau$ is the flare rise timescale. It has been shown earlier that all TFD variations can be modelled to surprising accuracy with a small number of flares consisting of an exponential rise, sharp peak and exponential decay superposed on a constant baseline flux (Valtaoja et al. 1999). This decomposition (described in more detail by Valtaoja et al. 1999) helps us to identify and isolate individual events as well as to estimate amplitudes and timescales of the outbursts.

Next we plotted the TFD decompositions and the flux variations of the VLBI components for each source. Two examples illustrate the results of this comparison: $1633+382$ (4C 38.41) and PKS 2230+114 (CTA 102) are shown in Figs. 1 and 2. The component identifications can be found in Jorstad et al. (2001a). Even at first glance, it is evident that there is a clear connection between the millimetre continuum variations and the VLBI component fluxes. Whenever there are enough VLBA observations, the summed flux curve of the VLBI components is similar to the continuum flux curve; only the amplitude of the former is $\sim 90 \%$ that of the latter. This is expected
Table 2. The percentage of each class of the radio-loud AGN in three different samples. $[\mathrm{HPQ}=$ high optical polarization quasar, $\mathrm{LPQ}=$ low optical polarization quasar, $\mathrm{BLO}=\mathrm{BL}$ Lacertae object, GAL $=$ radio galaxy and N/A = No exact classification available].

\begin{tabular}{lccc}
\hline \hline Class & Our sample & 2 Jy sample & EGRET blazars \\
\hline HPQ & $44 \%$ & $28 \%$ & $37 \%$ \\
LPQ & $26 \%$ & $32 \%$ & $30 \%$ \\
BLO & $26 \%$ & $28 \%$ & $26 \%$ \\
GAL & $4 \%$ & $8 \%$ & $0 \%$ \\
N/A & $0 \%$ & $4 \%$ & $7 \%$ \\
\hline
\end{tabular}

with the missing $10 \%$ of the flux in the VLBI maps probably just due to the insensitivity of high-frequency VLBI to diffuse emission. There is a slight time shift between the $37 \mathrm{GHz}$ TFD curves and the $43 \mathrm{GHz}$ VLBI component flux curves. This is understandable according to the shock models, since the maximum amplitude of the flare moves from high frequencies to lower frequencies as the shock evolves.

A much more interesting result is that for every superluminal ejection seen in the VLBA data, the TFD decomposition shows a coinciding flare. We examine ejections having zero epochs after the year 1990. For most of our sources, Metsähovi TFD monitoring is rather sparse before this and therefore not suitable for our comparison. We exclude two ejections because of large gaps in the Metsähovi flux curve at their zero epochs (the observation gap in 1994). We require that there be at least three observations of the ejected component and that the 

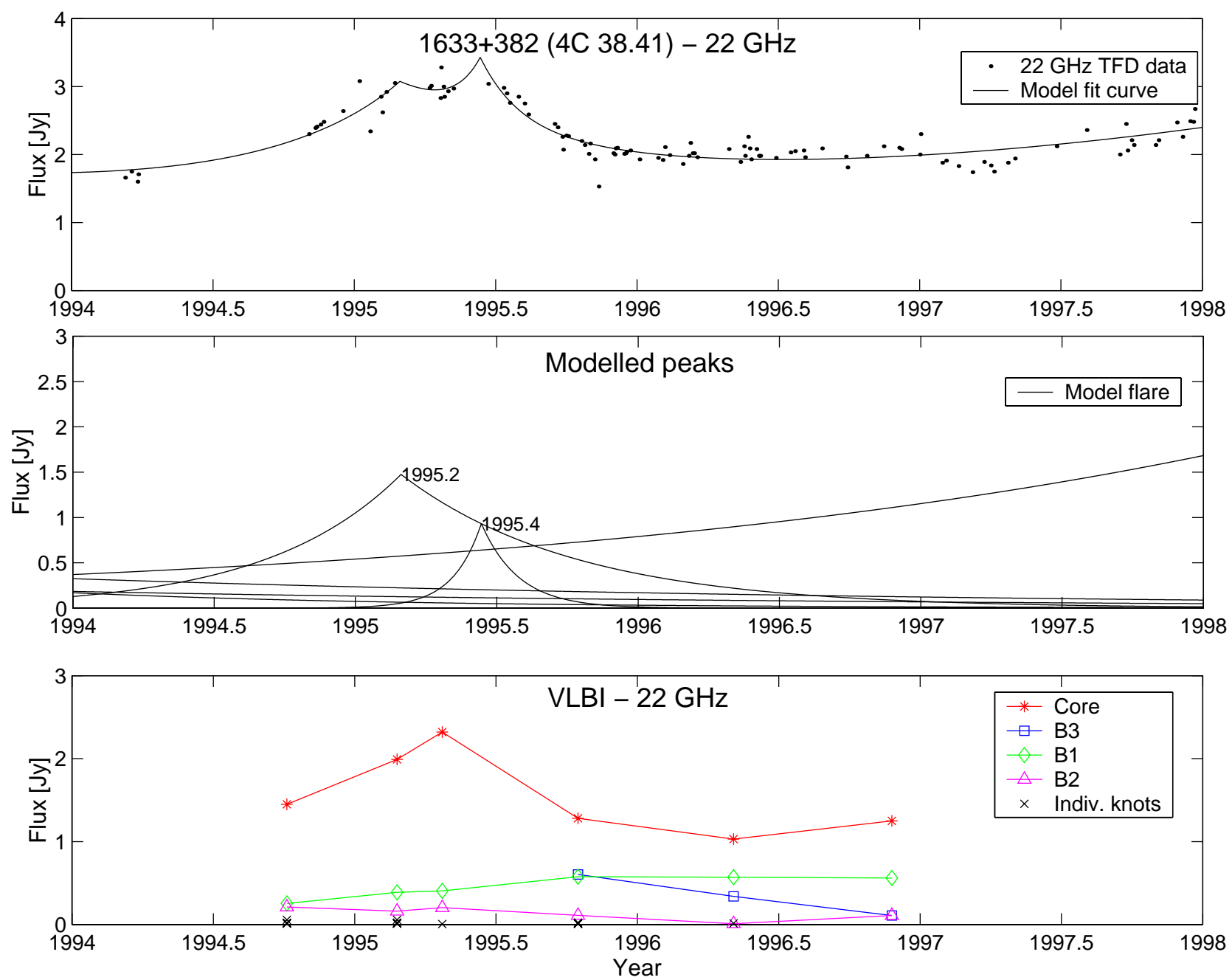

Fig. 1. An example (quasar 1633+382) of a graph containing TFD measurements, exponential flare model fits, and individual VLBI component flux density vs. time. The top panel presents total flux density observations at $22 \mathrm{GHz}$ from the Metsähovi quasar monitoring program (dots) and our fit (curve), which is a sum of individual exponential model flares superposed on a constant baseline flux. The modelled flares are shown in the middle panel with the epochs of the maximum flux density indicated. The bottom panel displays the flux evolution of the VLBI components at $22 \mathrm{GHz}$ from the VLBA observations by Jorstad et al. (2001a), whose component designations we adopt; the errors in flux density of the VLBI components are approximately $0.05 \mathrm{Jy}$.

observed flux density of the component be greater than $0.1 \mathrm{Jy}$ (the approximate noise level of Metsähovi observations) at some time. In our data, there are 29 ejections of VLBI components fulfilling the above criteria (see Table 3). The TFD flares corresponding to these 29 ejections are identified by comparing the component ejection times with the beginning times of the TFD flares, as well as by comparing the light curves of the VLBI components with those of the decomposed TFD flares.

We define the beginning of an exponential TFD flare as $t_{0, \mathrm{TFD}}=t_{\max }-\tau$, where $\tau$ is the variability timescale (e-folding time). This definition gives the point where $S\left(t_{0, \mathrm{TFD}}\right)=\frac{S_{\max }}{e}$. While there is no mathematical sense in defining the beginning of an exponential function, in reality there must be a starting point to a flare. We could instead estimate the beginning of the flare as the previous local minimum of the flux curve $\left(t_{\mathrm{lm}}\right)$. If we compare $t_{\mathrm{lm}}$ to $t_{0, \mathrm{TFD}}$ for an outburst that starts just after a local minimum, we see that the average time difference between the two is 0.0 years with a standard deviation of 0.4 years (see Fig. 3). Therefore, the average values of $t_{0, \mathrm{TFD}}$ and $t_{\mathrm{lm}}$ are the same. When two or more closely spaced outbursts blend together, the local minimum is no longer a good indicator of the start of the flare. In such a case the local minimum is near the peak rather than the beginning of the later flare. Hence, $t_{0, \mathrm{TFD}}$ is a more reliable and practical starting point to a flare.

We compare the extrapolated ejection epochs of the superluminal knots (from Jorstad et al. 2001a) with the beginning times of the TFD flares. In 28 of the 29 cases we find a TFD flare that occurred within $0.5 \mathrm{yr}$ of the ejection epoch. The only exception is component $\mathrm{E} 2+\mathrm{B} 1$ of $3 \mathrm{C} 279$, for which $t_{0, \mathrm{VLBI}}$ is not very well determined.

The frequency of large TFD flares $\left(\Delta S>0.3 \cdot S_{\text {quiescent }}\right)$ estimated from the Metsähovi data is 1 per 1.6 years. On the other hand, the frequency of observed superluminal ejections is approximately 1 per 2.3 years. Using these values we calculate the probability that a superluminal ejection could occur by random chance within a time interval $\mathrm{d} t$ before or after the 

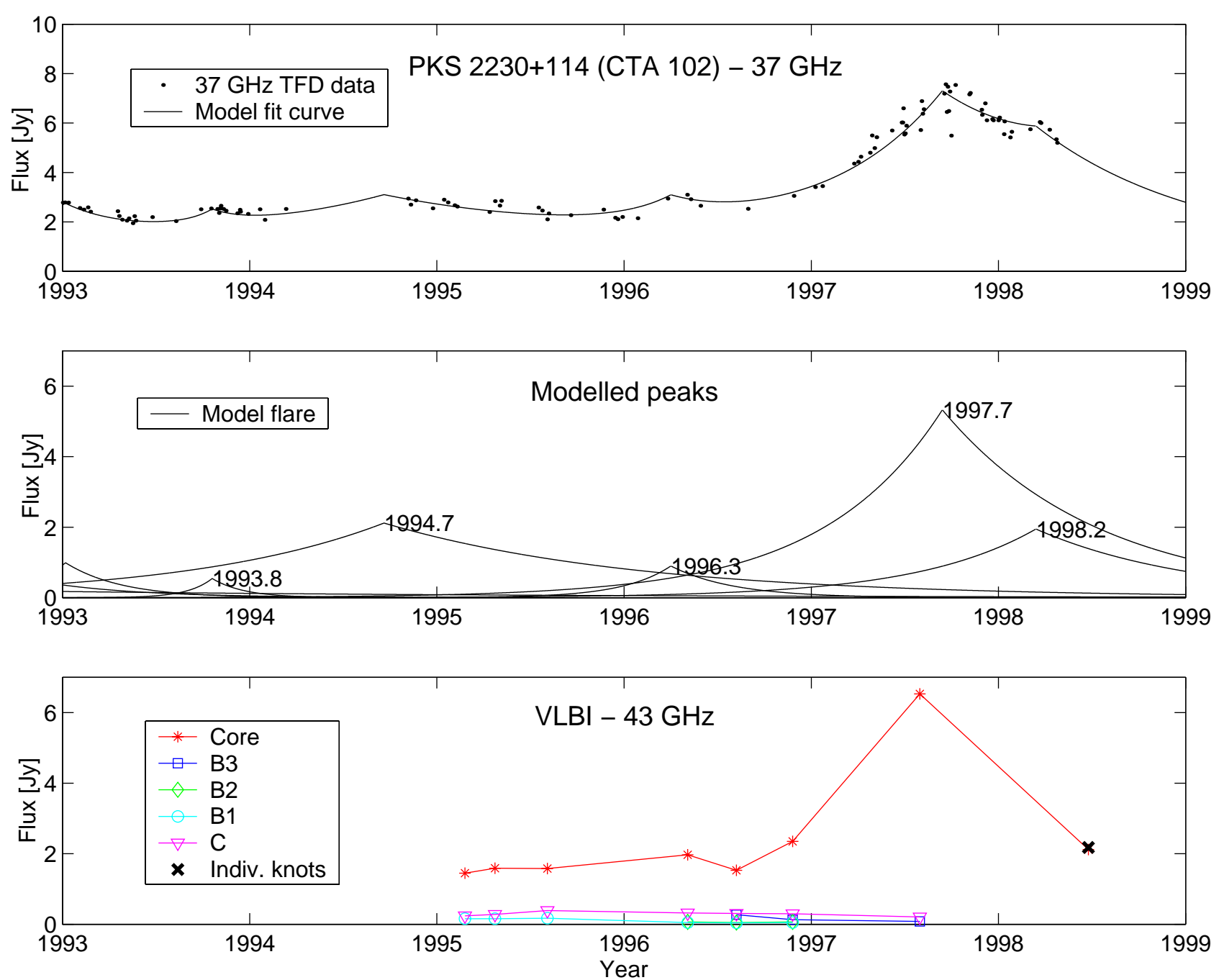

Fig. 2. Another sample TFD curve, exponential flare model fits, and individual VLBI component TFD values as a function of time. The 1998.48 data is from VLBA observations by Fredrik Rantakyrö (Rantakyrö et al. 2002). See the caption of Fig. 1 for details.

beginning of the TFD flare. The results are given in Table 4 . For every applied $\mathrm{d} t$ range the expected number of random occurrences is clearly much lower than the observed number of coincidences. The probability that 28 out of 29 ejections would be observed to occur randomly within $0.5 \mathrm{yr}$ of the beginnings of TFD flares is $<10^{-7}$. Hence, the correspondence between $t_{0, \mathrm{VLBI}}$ and $t_{0, \mathrm{TFD}}$ is real at a very high level of significance.

We therefore find that, at high radio frequencies, the start of a TFD flare precedes the arrival of a new superluminal knot at the position of the brightness centroid of the core of the jet. However, we do not have enough VLBI data to say if the converse is true, i.e., whether there is a new VLBI component for every TFD flare. When we see the new VLBI component for the first time, the flux of the TFD flare is usually already decreasing. This behaviour is analysed in Sect. 4, in which we discuss the so-called core flares.

The mean time difference between the zero epoch of the VLBI components and the beginning of the TFD flares $\Delta t=t_{0, \mathrm{VLBI}}-t_{0, \mathrm{TFD}}$ is $+(0.19 \pm 0.04) \mathrm{yr}$ (ignoring component E2+B1 in 3C 279). The extrapolated ejection time of a VLBI component is therefore $\sim 0.2 \mathrm{yr}$ after the beginning of the associated TFD flare, on average. This may indicate that the proper motion of a typical VLBI knot accelerates during the early stages in the component's evolution. On the other hand, we note that $t_{0, \mathrm{VLBI}}$ is the moment when the component is coincident with the brightness centroid of the core. In this case, the TFD flare might begin when the disturbance that creates the shock first hits the inner edge of the core, which would occur before $t_{0, \mathrm{VLBI}}$.

The fluxes of the VLBI components and the decomposed TFD flares are correlated. In Fig. 4 we plot the VLBI component fluxes vs. the decomposed TFD flare fluxes at the VLBI epochs (from our exponential-flare model fits). The Spearman correlation coefficient of this graph is $r_{\mathrm{S}}=0.817$; the probability that $r_{\mathrm{S}}$ would be this high from uncorrelated data $\sim 10^{-14}$. The linear Pearson correlation coefficient $r_{\mathrm{P}}=0.76$, which corresponds to a probability of $\sim 10^{-12}$ that the correlation is by chance. Furthermore, in $59 \%$ of the cases the fluxes differ by less than a factor of two. There is therefore a clear connection between new VLBI components in the jet and mm-wave flares in $\gamma$-ray blazars. 
Table 3. The zero epochs of the VLBI components $\left(t_{0, \mathrm{VLBI}}\right)$ determined by Jorstad et al. (2001a) and the start times of the corresponding TFD flares $\left(t_{0, \mathrm{TFD}}\right)$. The last column gives the time difference $\Delta t=t_{0, \mathrm{VLBI}}-t_{0, \mathrm{TFD}}$. The designation of the components follows Jorstad et al. (2001a).

\begin{tabular}{lcccc}
\hline \hline Source & Comp. & $t_{0, \mathrm{VLBI}}$ & $t_{0, \mathrm{TFD}}$ & $\Delta t$ [yr] \\
\hline $0202+149$ & B & $1994.8 \pm 0.1$ & 1994.7 & 0.1 \\
$0219+428$ & B4 & $1995.7 \pm 0.4$ & 1995.7 & 0.0 \\
& B3 & $1995.4 \pm 0.1$ & 1994.9 & 0.5 \\
$0235+164$ & B1 & $1995.1 \pm 0.2$ & 1994.8 & 0.3 \\
$0420-014$ & B & $1995.3 \pm 0.1$ & 1994.9 & 0.4 \\
$0458-020$ & B2 & $1994.0 \pm 0.1$ & 1993.6 & 0.4 \\
$0528+134$ & B4 & $1995.5 \pm 0.1$ & 1995.5 & 0.0 \\
& B3 & $1994.8 \pm 0.1$ & 1994.7 & 0.1 \\
& B2 & $1994.5 \pm 0.2$ & 1994.2 & 0.3 \\
$0827+243$ & B1 & $1993.4 \pm 0.7$ & 1993.4 & 0.0 \\
$0851+202$ & B1 & $1994.7 \pm 0.1$ & 1994.6 & 0.1 \\
& B3 & $\approx 1996.6$ & 1996.6 & 0.0 \\
$1156+295$ & B2 & $1995.6 \pm 0.1$ & 1995.6 & 0.0 \\
& B3 & $1996.3 \pm 0.2$ & 1996.0 & 0.3 \\
$1226+023$ & B2 & $1995.3 \pm 0.1$ & 1995.1 & 0.2 \\
& B5 & $1993.4 \pm 1.3$ & 1993.4 & 0.0 \\
& B3 & $1992.4 \pm 0.3$ & 1991.9 & 0.5 \\
& B2 & $1991.3 \pm 0.4$ & 1991.0 & 0.3 \\
$1253-055$ & B1 & $1990.4 \pm 0.3$ & 1990.7 & 0.3 \\
& B3 & $1995.7 \pm 0.1$ & 1995.5 & 0.2 \\
& B2 & $1994.2 \pm 0.2$ & 1993.8 & 0.4 \\
& E2+B1 & $1993.5 \pm 0.2$ & 1992.4 & 1.1 \\
$1510-089$ & B1 & $1996.1 \pm 0.1$ & 1996.3 & -0.2 \\
& D2 & $1994.1 \pm 0.2$ & 1993.9 & 0.2 \\
$1633+382$ & B3 & $1994.8 \pm 0.1$ & 1994.7 & 0.1 \\
$2230+114$ & B3 & $1996.1 \pm 0.1$ & 1996.0 & 0.1 \\
& B1 & $1994.3 \pm 0.1$ & 1993.9 & 0.4 \\
& B3 & $1995.6 \pm 0.2$ & 1995.4 & 0.2 \\
& B2 & $1995.1 \pm 0.1$ & 1995.0 & 0.1 \\
\hline & & & &
\end{tabular}

Table 4. The probability $P$ of coincidence of superluminal ejections and the start of TFD flares, for both the random and observed case $\left(P_{\text {Observed }}=N_{\text {Associated }} / N_{\text {Total }}\right)$. The interval $\mathrm{d} t$ is the maximum time difference between the ejection of the knot and the beginning of a TFD flare for them to be considered associated. $\rho$ is the probability that the observed number of associations could occur by random chance.

\begin{tabular}{cccc}
\hline \hline $\mathrm{d} t[\mathrm{yr}]$ & $P_{\text {Random }}$ & $P_{\text {Observed }}$ & $\rho$ \\
\hline 0.5 & $47 \%$ & $97 \%$ & $<10^{-7}$ \\
0.4 & $40 \%$ & $90 \%$ & $<10^{-7}$ \\
0.3 & $32 \%$ & $76 \%$ & $<10^{-6}$ \\
0.2 & $22 \%$ & $59 \%$ & $<10^{-4}$ \\
0.1 & $12 \%$ & $41 \%$ & $<10^{-5}$ \\
\hline
\end{tabular}

\section{VLBI core flares}

In our sample, the core is the brightest VLBI component in 152 of the 165 VLBA images. It usually contains $50-90 \%$ of the total flux density of the source. Typically it is the core region that becomes brighter and fainter during the TFD flares. Similar behaviour has been noted previously in, for example, 3C 279 (Wehrle et al. 2001). This pattern is completely different from that observed in $3 \mathrm{C} 345$, in which the fluxes of the components peak well downstream of the core

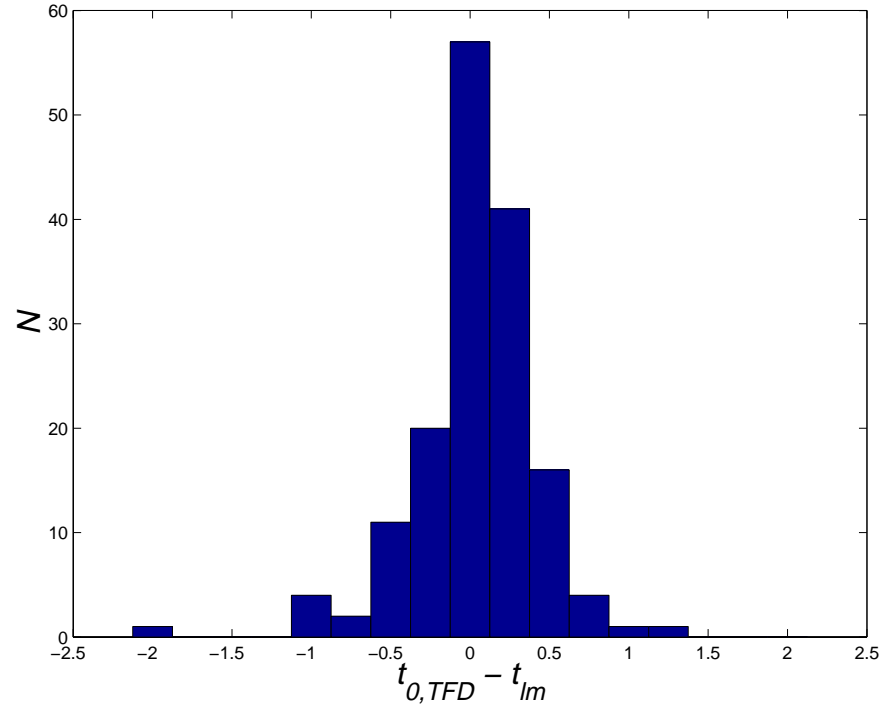

Fig. 3. The time difference between $t_{0, \mathrm{TFD}}=t_{\max }-\tau$ and the epochs of the local minima in our data.

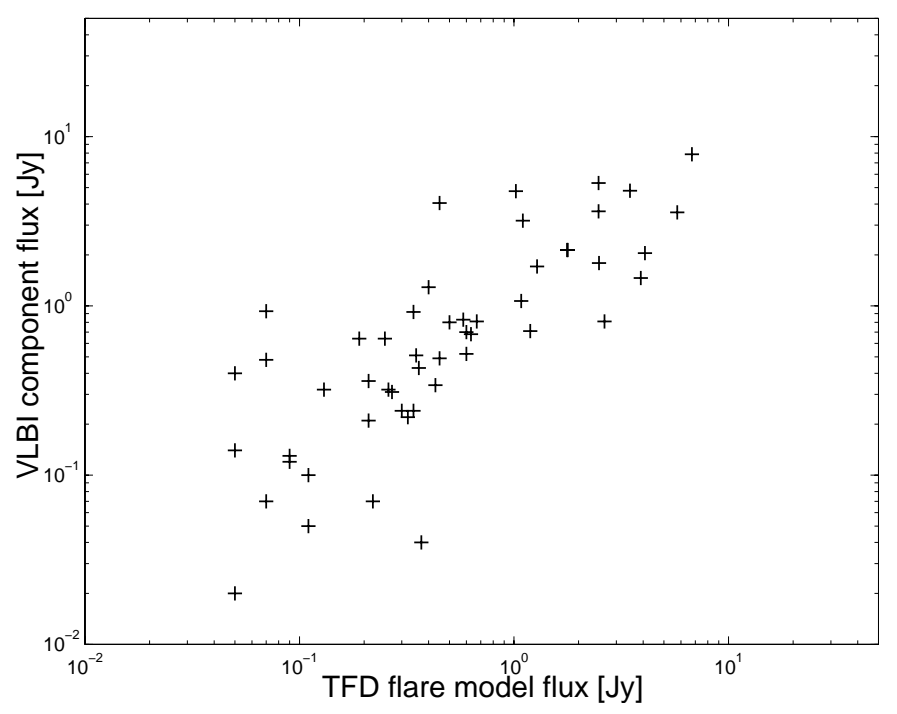

Fig. 4. Decomposed TFD flare model fluxes compared with associated VLBI component fluxes at the time of the VLBI observations.

(Valtaoja et al. 1999). In our sample there is one source, PKS $0202+149$, with a component (B) whose flux clearly peaks downstream. However, this component is not very strong $(0.3$ Jy at maximum) and it still peaks quite close to the core at a separation of only 0.36 mas. There are also a few other sources in our sample with a component that might peak downstream, but we do not have sufficiently well-sampled observations to confirm these. In any case, these features are considerably weaker than the flux level involved in most of the core flares.

These apparent VLBI core flares are therefore largely responsible for the TFD variability in our sources. The question is: Are these core flares due to true changes in the radio core (changes in bulk Lorentz factor, electron energy spectrum, etc.) or are they associated with the birth of new moving VLBI components that blend with the core at the resolution of the images? 
We define a core flare to be an increase in the VLBI coreregion flux that is (1) $>30 \%$ within one year and (2) greater than the noise level of the TFD curve. There were altogether 24 core flux variations satisfying our conditions. When analysing these VLBI core flares and coincident TFD variations, we noticed that, in 11 cases, after the core flare and an associated TFD outburst had peaked and were already fading, a new VLBI component appeared in the jet (see Table 5). Moreover, the flux of this new component was decreasing as well. Of 13 cases not showing a new component after the core flare, 6 simply did not have enough data because the core flare occurred during the last observing epoch. Thus, in 11 of the 18 cases $(61 \%)$ the observed increase in VLBI core flux is consistent with being caused by the appearance of a new moving component, which brightens and then starts to decay within $\approx 0.15$ mas (the typical VLBA beamsize at $43 \mathrm{GHz}$ ) of the actual radio core. This represents a high percentage if we consider the sparsity of the VLBI time coverage, systematic uncertainties in VLBI model fitting, and the problems related to component identification across epochs.

We explore the above interpretation further by reconstructing the core flares under the assumption that only new components contribute to the variations in flux while the brightness of the actual core remains constant. We then decompose the total flux into a constant baseline flux from the core plus a new shock component with variable flux. We choose the value of the baseline flux density to be the lowest observed level (either the minimum core-region flux or the weakest TFD flux, whichever is lower). Two examples of our reconstructions are presented in Figs. 5 and 6 (PKS 0420-014 and PKS 0528+134).

In order to test the above scenario, we estimate the separation between the true core and the new VLBI component during core flares by assuming that the proper motions remain constant from the birth of a knot to its last appearance on the images of Jorstad et al. (2001a). We find five cases (PKS 0420-014: 1995 Apr. 22, 1995 Jun. 21, 1995 Aug. 04; PKS 0528+134: 1995 Apr. 22, and 1156+295: 1996 May 04) in which the expected separation during a VLBA observation was larger than about one-third of the beam size. If the source is not very complicated, i.e., if it can be described using a small number of circular-Gaussian components, partially resolved compact structure is revealed by non-zero closure phases on the longest baselines. Closure phases are constructed to cancel out antenna-based calibration errors, hence they indicate the presence of non-point components in a model-independent fashion.

Partially resolved structure in the core region can take on two basic forms: (1) a slightly extended core with no other components or (2) one or more non-core components within one beamwidth of the brightness centroid of the core. To compare these two hypotheses we plot closure phases from triangles consisting of both long and short baselines (Figs. 7 and 8). We superimpose two best-fit models corresponding to the above hypotheses. It is evident - and also expected - that these models differ significantly only on long baselines. It can be clearly seen that the two-component core models provide much better fits to the data than do the single-component models. Also, in Fig. 7 the progressively increasing absolute value of the closure phase over the three successive epochs can be explained by a moving component close to the core.
We therefore find evidence for new components in the vicinity of the VLBI core during major TFD flares, but the brightness of these moving knots has usually decayed considerably by the time the features become distinct from the core on VLBI maps. This implies that a majority - perhaps all - of the strongest TFD flares are associated with bright knots that fade within $\sim 0.1-0.3$ mas of the core. (The only source in our data set that has substantial variations in flux outside the core region is $3 \mathrm{C} 454.3$, where stationary feature $\mathrm{C}$ has brightness and variability comparable to the core.) If the moving components are shock waves, the shocks must therefore develop much more quickly, and closer to the core, than previously thought. The bright features found in jets of classical superluminal radio sources well downstream of the core are mainly lowerfrequency phenomena, although in some objects (e.g., 3C 279; see Wehrle et al. 2001) an occasional prominent component remains bright long after it detaches from the core on the images. The implication is that the critical synchrotron frequency of the highest energy electrons in the knot decays rapidly beyond the core region. This is probably the combination of (1) radiative energy losses near the core, (2) decay of the magnetic field and electron energies from downstream expansion of the jet, and (3) weak in situ particle acceleration outside the core region. These conditions have important implications for models of shocks and other structures in relativistic jets.

It is possible that all of the variations in flux in the VLBI core region during these flares actually occur in new moving knots. However, our observations are also consistent with the possibility that a disturbance both creates a new shock wave and brightens the core itself as it passes through the core region. There may be some evidence supporting this scenario, since in two sources, 3C 279 and 1510-089, the new VLBI component is observed during (rather than only after) a major core flare. Based on the present data, we cannot determine which scenario is correct. The model in which the TFD variability is completely due to shocks is the simpler and requires fewer assumptions. On the other hand, it would be surprising (and revealing in terms of models for the core; see Daly \& Marscher 1988) if the disturbance in velocity and/or energy density that creates the shock did not also affect the core (see, e.g., the hydrodynamical simulations of Gómez et al. 1997).

We calculate the distance from the core where a new component reaches its maximum luminosity:

$l=\Gamma D \frac{\Delta t_{\mathrm{obs}}}{1+z} \beta c$,

where $l$ denotes the distance from the core along the jet, $\Gamma$ is the bulk Lorentz factor, $D$ is the Doppler factor, $\Delta t_{\text {obs }}$ is the observed timescale for a knot to reach maximum luminosity, $z$ is the redshift, $\beta=v / c \approx 1, v$ is the bulk velocity of the component and $c$ is the velocity of light. We derive the Doppler factor $D$ from the variability data using the procedure described in Lähteenmäki \& Valtaoja (1999). We then determine the Lorentz factor $\Gamma$ from this value of $D$ together with the apparent superluminal speed of the associated VLBI component (Jorstad et al. 2001a). The rise time $\Delta t_{\text {obs }}$ was determined as the difference between the epoch of the TFD flare peak and the extrapolated zero epoch of the associated VLBI component, 

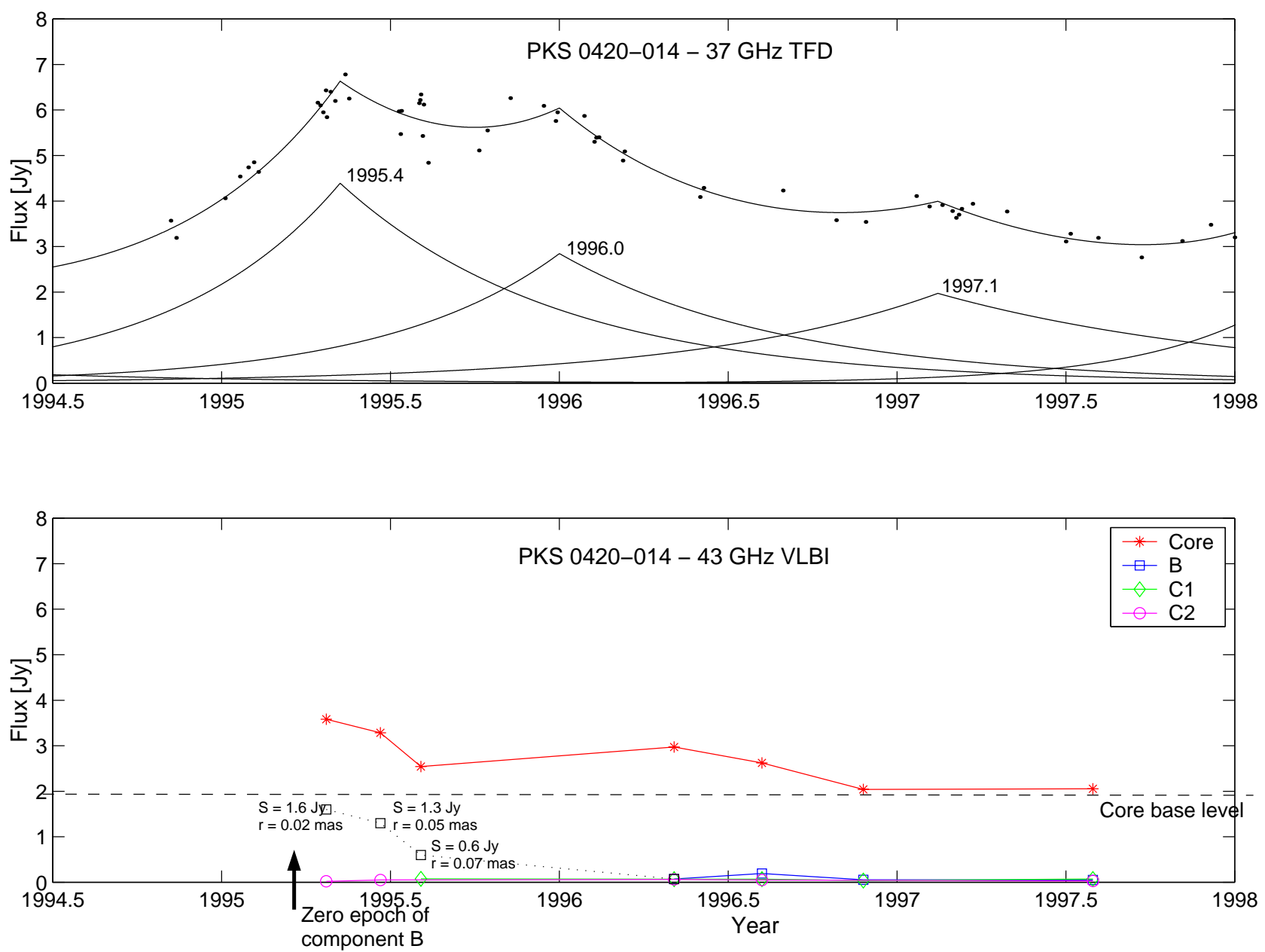

Fig. 5. Reconstruction of the "core flare" in quasar PKS 0420-014. The upper panel shows the $37 \mathrm{GHz}$ light curve, three exponential flare models, and the summed model fit to the TFD. The lower panel presents the light curves of the individual VLBI components. The VLBI core flare in 1995 is decomposed into a core component with a constant flux (horizontal dashed line) and a new component with decreasing flux (dotted curve). The figure also displays the estimated fluxes and separations from the core for component B of Jorstad et al. (2001a). The arrow gives the estimated epoch of zero separation from the core, obtained by extrapolation of the VLBI data.

hence $\Delta t_{\mathrm{obs}}=t_{\mathrm{max}, \mathrm{TFD}}-t_{0, \mathrm{VLBI}}$. The mean observed timescale for shock evolution $\Delta t_{\mathrm{obs}}$ is $140_{-115}^{+135}$ days, which gives an average value for $l$ of $6 \pm 3$ pc along the jet at $43 / 37 \mathrm{GHz}$ for our sample. A timescale of 140 days corresponds on average to a movement of $\approx 0.11$ mas in our sample (the average proper

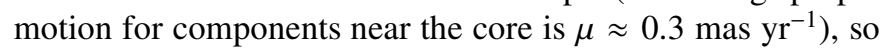
that the components usually reach their maximum brightness well within the beamsize of the VLBA.

\section{Conclusions}

Our comparisons between VLBI and TFD data show that there is a clear connection between TFD outbursts of blazars and structural changes in their jets. For every new VLBI component emerging into the jet, there is a coincident TFD flare, and also the extrapolated zero epoch of the VLBI component agrees well with the beginning of the TFD flare. At a later stage, there is a clear correspondence between the flux density of the VLBI component and that of the modelled exponential TFD flare. We conclude that one can - for most events - use the beginning of a major TFD flare as an indicator of the time of ejection of a VLBI component.
The most pronounced TFD outbursts seem to occur within the innermost few tenths of a milliarcsecond of the core, which is comparable to the maximum resolution of present-day VLBI. The flares are associated with the production of new superluminal components, commonly interpreted as shocks. However, with the present observations, we cannot determine definitively how much of the flare is contained in the shock and how much is due to changes in the flux of the core itself as it reacts to the disturbance that creates the shock wave.

Due to insufficient resolution even at $43 \mathrm{GHz}$, we usually see only "core flares" in our sources. An example of the effects of limited resolution is BLO 1749+096. Despite violent variability in TFD, this object is virtually unresolved even with a beamsize of 0.6 mas along the jet in global $22 \mathrm{GHz}$ VLBI observations (Wiik et al. 2001). Only global $86 \mathrm{GHz}$ VLBI, with a 0.22 mas beam, has been able to resolve the jet (Lobanov et al. 2000).

Based on the results obtained in Sect. 4, we suggest that every large TFD outburst results from a new shock being created in the jet, and not just from a change in the parameters of the ambient jet such as the bulk Lorentz factor or the 

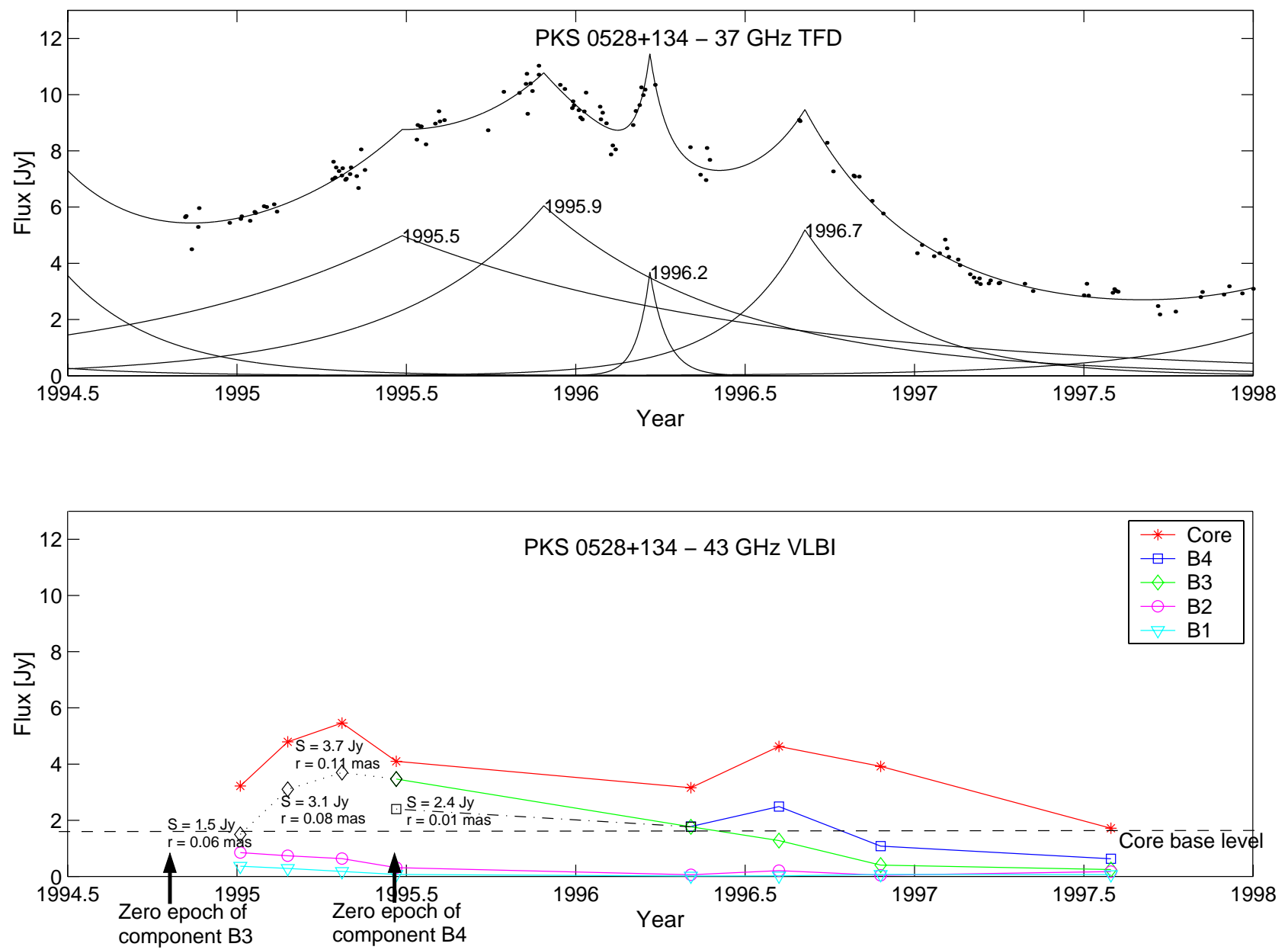

Fig. 6. A reconstruction of the "core flares" in quasar PKS 0528+134. That corresponding to the TFD flare in 1995.5 and component B3 of Jorstad et al. (2001a) can be reconstructed easily (dotted line), but for the flare in 1995.9 (corresponding to component B4 of Jorstad et al. 2001a) we do not have enough VLBI observations to determine whether its behaviour is similar.

Table 5. A list of VLBI core flares with a new moving VLBI component appearing after the flare. The time span of the core flare is $t_{\mathrm{CF}}, \Delta S_{\mathrm{CF}}$ is the lower limit for the observed amplitude of the core flare, $t_{\mathrm{NC}}$ is the time when the new moving component is first detected, $S_{\mathrm{NC}}$ is its flux density, and $R$ is its distance from the core at this time.

\begin{tabular}{lcccccl}
\hline \hline Source & $v[\mathrm{GHz}]$ & $t_{\mathrm{CF}}$ & $\Delta S_{\mathrm{CF}}[\mathrm{Jy}]$ & $t_{\mathrm{NC}}$ & $S_{\mathrm{NC}}[\mathrm{Jy}]$ & $R[\mathrm{mas}]$ \\
\hline $0219+428$ & 43 & $1995.47-1996.60$ & 0.45 & 1996.60 & 0.22 & 0.51 \\
$0420-014$ & 43 & $1995.31-1995.59$ & 1.04 & 1996.34 & 0.09 & 0.21 \\
$0458-020$ & 43 & $1995.31-1995.47$ & 0.40 & 1995.47 & 0.60 & 0.20 \\
$0528+134$ & 22 & $1994.65-1995.01$ & 2.30 & 1995.01 & 1.69 & 0.19 \\
$0528+134$ & 43 & $1995.01-1995.47$ & 2.24 & 1995.47 & 3.47 & 0.14 \\
$0851+202$ & 43 & $1995.47-1996.34$ & 1.91 & 1996.34 & 0.28 & 0.36 \\
$1156+295$ & 22 & $1996.34-1996.60$ & 0.31 & 1996.60 & 0.34 & 0.20 \\
$1222+216$ & 22 & $1996.90-1997.58$ & 0.40 & 1997.58 & 0.09 & 0.45 \\
$1633+382$ & 22 & $1994.76-1995.79$ & 1.04 & 1995.79 & 0.60 & 0.13 \\
$2230+114^{*}$ & 43 & $1996.60-1998.48$ & 5.00 & 1998.48 & 2.14 & 0.11 \\
$2251+158$ & 43 & $1995.01-1995.31$ & 4.92 & 1995.31 & 4.43 & 0.06 \\
\hline
\end{tabular}

* Includes 1998 VLBA data from Rantakyrö et al. (2002).

electron energy spectrum. The essential difference between these two models is that, in the jet-parameter model, luminosity changes in the underlying flow cause the observed flux variability, whereas in the shock model the flares represent an evolving shock wave. The core itself can also become brighter if the shock wave disturbs it when passing through the nozzle of the jet. However, this does not change our suggestion that there is a shock associated with every large TFD flare. This has important implications for models of $\gamma$-ray emission from blazars (see, e.g., Valtaoja et al. 2002; Lähteenmäki \& Valtaoja 2002; Jorstad et al. 2001b). 
0420-014 (1995 Apr 22)
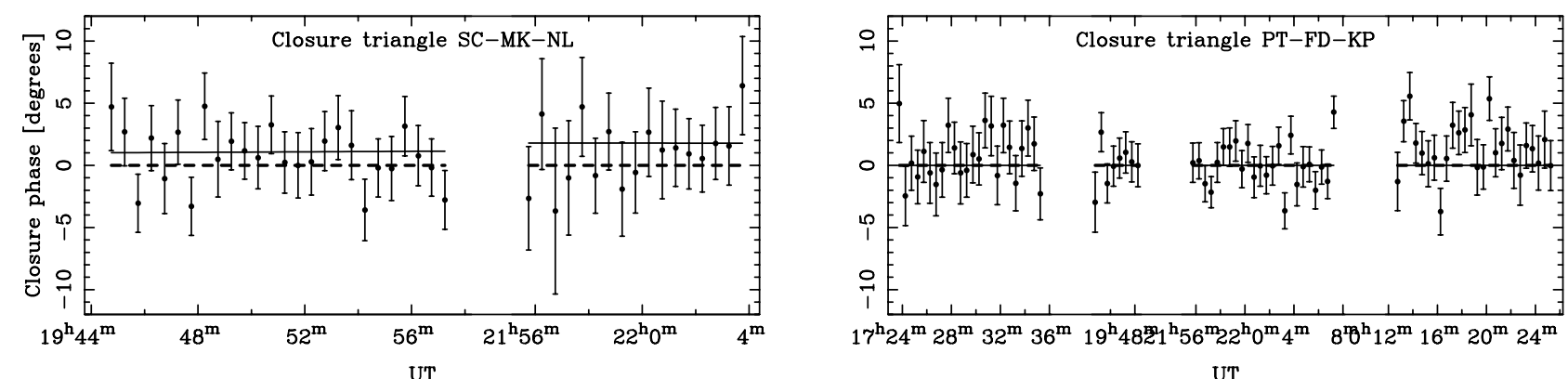

0420-014 (1995 June 21)
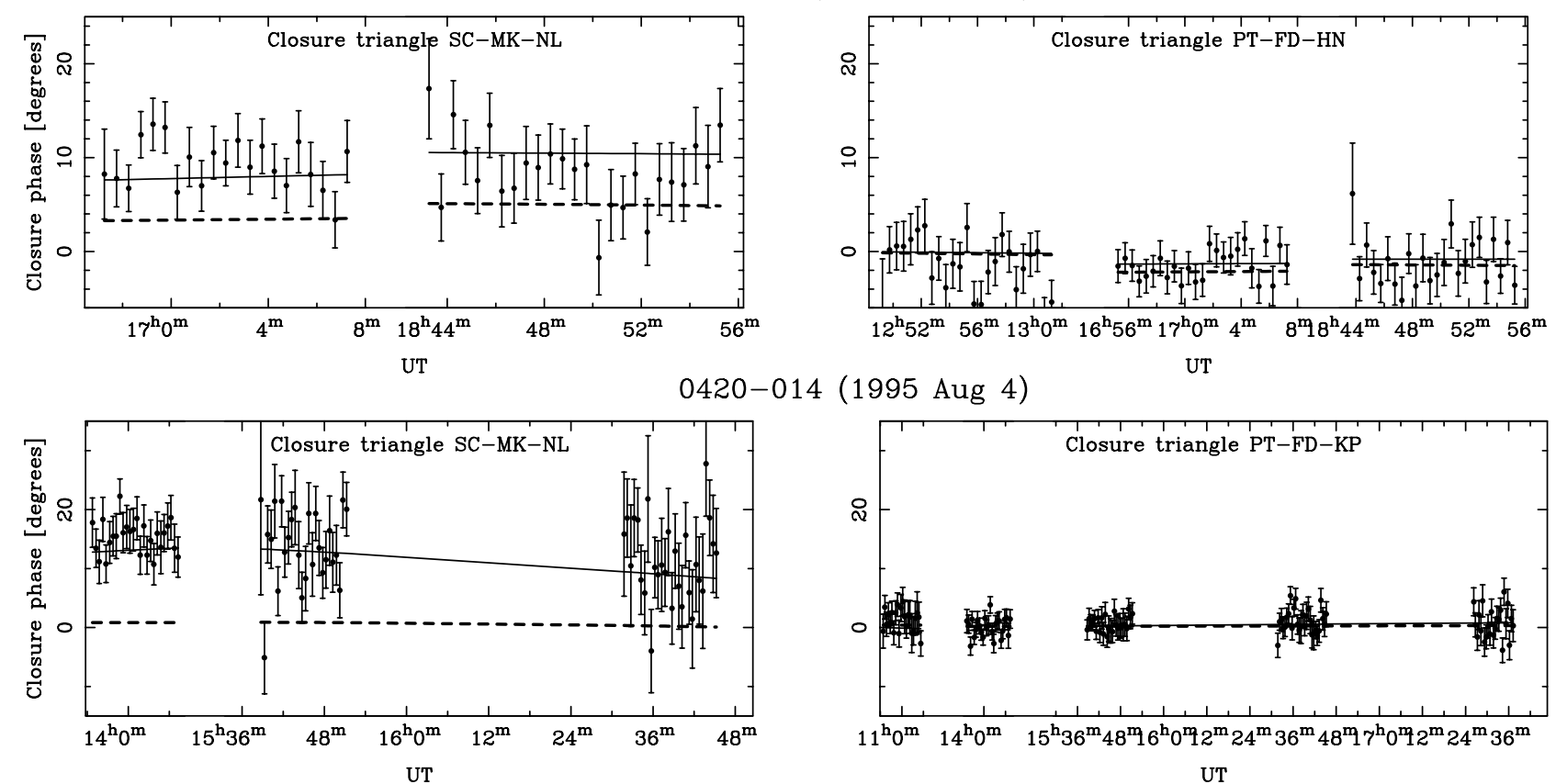

0420-014 (1997 Jul 31)
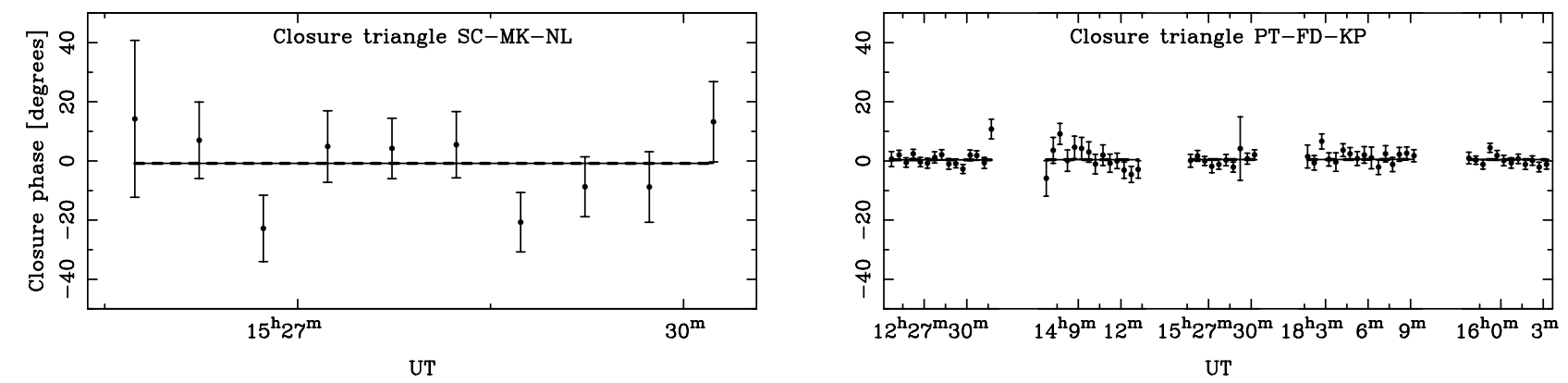

Fig. 7. Closure phases from triangles consisting of long (left panels) and short (right panels) baselines from a series of observations of PKS 0420-014. Two core-region models are superimposed: dashed curve: a single, perhaps extended component; solid curve: a point-like core plus a second point-like component. The fourth epoch (1997 July 31) is for comparison only: no component close to the core is expected from the TFD light curves nor indicated by the VLBA data.

Given the possible blending of a new shock component with the core on VLBI images, one has to be very careful when drawing conclusions based on the behaviour of the core. For example, Lobanov \& Zensus (1999) have studied the spectral evolution of the jet components in quasar 3C 345. They find two time intervals during which they could not reproduce the observed spectral changes (frequency of the spectral maximum was rising while the flux density at this frequency was constant or even decreasing slightly) of the core component in terms of the relativistic shock model. However, their spectral fits included only a single component in the core region. According to our results, there is likely to be a second, variable component blending with the core that can cause the observed spectral changes. We suggest that it may be possible to explain the behaviour of the core in a way which is consistent with the relativistic shock model.

The results presented in this article indicate that VLBI observations at $90 \mathrm{GHz}$ are needed in order to study new moving 

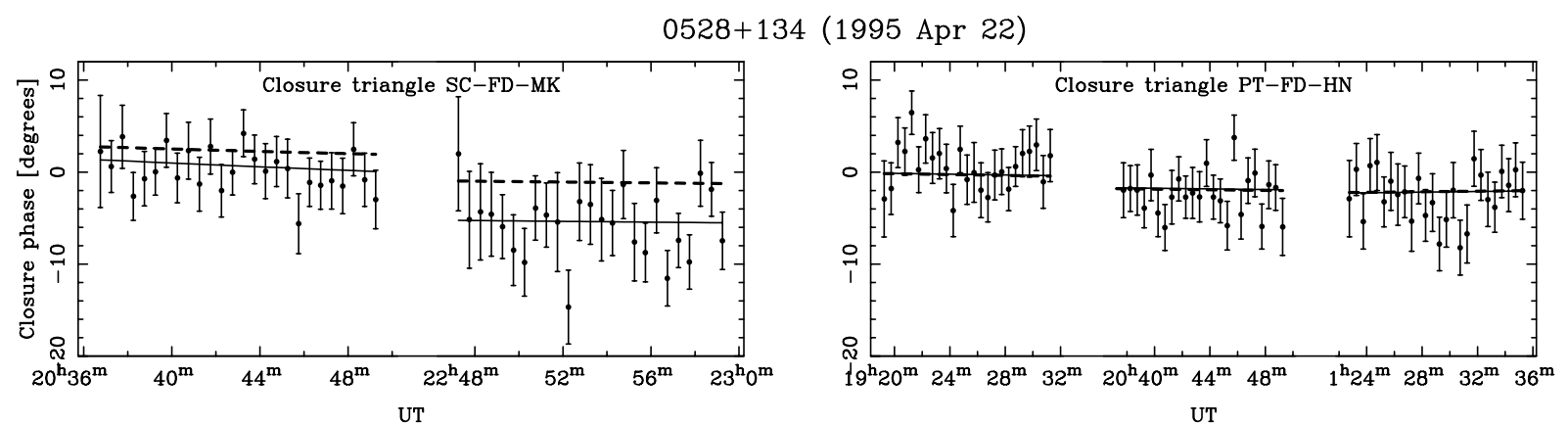

$1156+295$ (1996 May 4)
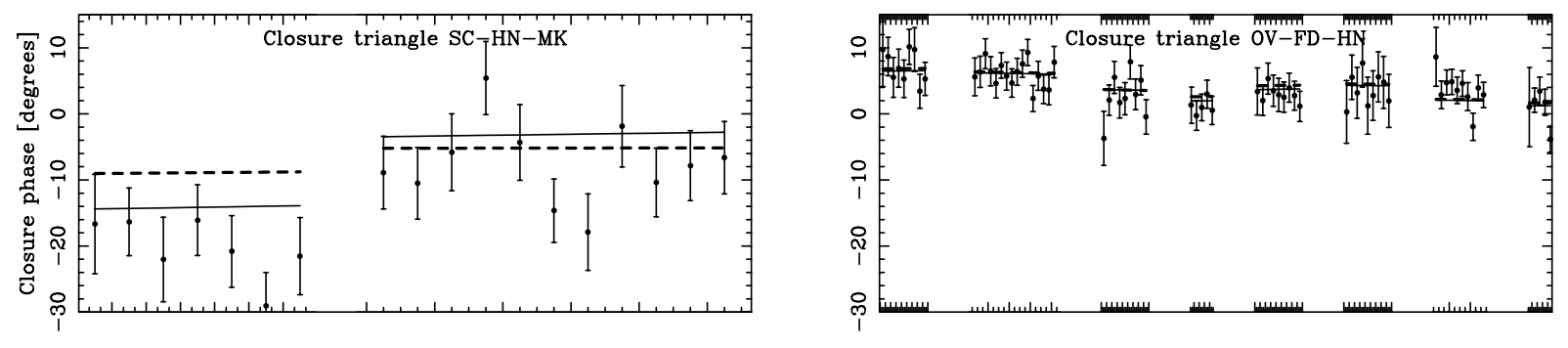

UT

UT

Fig. 8. Closure phases from observations of $0528+134$ and 1156+295. Continued from Fig. 7.

components during the stage when they are still growing, and still greater resolution is required to see the actual formation of the shock. This type of study would benefit greatly from future space interferometry missions such as the proposed VSOP-2 and iARISE missions. In addition to finer resolution, we also need better sampled VLBI component flux curves, which can be obtained from intensive VLBI monitoring.

Acknowledgements. We thank Fredrik Rantakyrö for providing us the VLBA observations of CTA 102 prior to publication. This work was supported in part by NASA through CGRO Guest Investigator grants NAG5-7323 and NAG5-2508, and by U.S. National Science Foundation grants AST-9802941 and AST-0098579. The VLBA is a facility of the National Radio Astronomy Observatory, operated by Associated Universities, Inc., under cooperative agreement with the U.S. National Science Foundation.

\section{References}

Abraham, Z., Carrara, E. A., Zensus, J. A., \& Unwin, S. C. 1996, A\&AS, 115, 543

Bartel, N., Herring, T. A., Ratner, M. I., Shapiro, I. I., \& Corey, B. E. 1986, Nature, 319, 733

Björnsson, C.-I., \& Aslaken, T. 2000, ApJ, 533, 787

Britzen, A., Witzel, A., Krichbaum, T. P., et al. 2000, A\&A, 360, 65

Daly, R. A., \& Marscher, A. P. 1988, ApJ, 334, 539

Gómez, J. L., Martí, J. M., Marscher, A. P., Ibáñez, J. M., \& Alberdi, A. 1997, ApJ, 482, L33

Hartman, R. C., Bertsch, D. L., Bloom, S. D., et al. 1999, ApJS, 123,79

Hughes, P. A., Aller, H. D., \& Aller, M. F. 1985, ApJ, 298, 301

Hughes, P. A., Aller, H. D., \& Aller, M. F. 1989a, ApJ, 341, 54

Hughes, P. A., Aller, H. D., \& Aller, M. F. 1989b, ApJ, 341, 68

Hughes, P. A., Aller, H. D., \& Aller, M. F. 1991, ApJ, 374, 57

Jorstad, S. G., Marscher, A. P., Mattox, J. R., et al. 2001a, ApJS, 134, 181
Jorstad, S. G., Marscher, A. P., Mattox, J. R., et al. 2001b, ApJ, 556, 738

Kellermann, K. I., \& Pauliny-Toth, I. I. K. 1969, ApJ, 155, L71

Krichbaum, T. P., Krauss, A., Otterbein, K., et al. 1998, Sub-mas Jets in Gamma-Active Blazars: Results from High Frequency VLBI, In IAU Colloquium 164: Radio Emission from Galactic and Extragalactic Compact Sources, ed. J. A. Zensus, G. B. Taylor, \& J. M. Wrobel, ASP Conf. Ser., 144, 37

Kühr, H., Witzel, A., Pauliny-Toth, I. I. K., \& Nauber U. 1981, A\&AS, 45,367

Lobanov, A. P., \& Zensus, J. A. 1999, ApJ, 521, 509

Lobanov, A. P., Krichbaum, T. P., Graham, D. A., et al. 2000, A\&A, 364,391

Lähteenmäki, A., \& Valtaoja, E. 1999, ApJ, 521, 493

Lähteenmäki, A., \& Valtaoja, E. 2002, in preparation

Marscher, A. P., \& Gear, W. K. 1985, ApJ, 298, 114

Marscher, A. P., Gear, W. K., \& Travis, J. P. 1992, Variability of Nonthermal Continuum Emission in Blazars, In Variability of Blazars, ed. E. Valtaoja, \& M. Valtonen (Cambridge University Press), 85

Mattox, J. R., Hartman, R. C., \& Reimer, O. 2001, ApJS, 135, 155

Mutel, R. L., Phillips, R. B., Su, Bumei, \& Bucciferro, R. R. 1990, ApJ, 352, 81

Rantakyrö, F. T., Wiik, K., Tornikoski, M., Valtaoja, E., \& Bååth, L. B. 2002, A\&A, submitted

Teräsranta, H., Tornikoski, M., Mujunen, A., et al. 1998, A\&AS, 132, 305

Türler, M., Courvoisier, T. J.-L., \& Paltani, S. 1999, A\&A, 349, 45

Valtaoja, E., Haarala, S., Lehto, H., et al. 1988, A\&A, 203, 1

Valtaoja, E., Lähteenmäki, A., \& Teräsranta, H. 1992a, A\&AS, 95, 73

Valtaoja, E., Teräsranta, H., Urpo, S., et al. 1992b, A\&A, 254, 71

Valtaoja, E., Lähteenmäki, A., Teräsranta, H., \& Lainela, M. 1999, ApJS, 120, 95

Valtaoja, E., Savolainen, T., Wiik, K., \& Lähteenmäki, A. 2002, PASA, 19, 117

Wehrle, A. E., Piner, B. G., Unwin, S. C., et al. 2001, ApJS, 133, 297

Wiik, K., Valtaoja, E., \& Leppänen, K. 2001, A\&A, 380, 72 